Marquette University

e-Publications@Marquette

Mechanical Engineering Faculty Research and

Publications

Engineering, College of

$1-1-2014$

Evaluation of Forearm Muscle Fatigue from

Operating a Motorcycle Clutch

Megan O. Conrad

Oakland University

Richard W. Marklin

Marquette University, richard.marklin@marquette.edu

Published version. Journal of Ergonomics (2014): S4:006. DOI. (C) 2014 Conrad MO, et al. This is an open-access article distributed under the terms of the Creative Commons Attribution License, which permits unrestricted use, distribution, and reproduction in any medium, provided the original author and source are credited. 


\title{
Evaluation of Forearm Muscle Fatigue from Operating a Motorcycle Clutch
}

\author{
Megan O. Conrad1* and Richard W. Marklin
}

${ }^{1}$ Department of Industrial and Systems Engineering, Oakland University, Rochester, Michigan, USA

${ }^{2}$ Department of Mechanical and Industrial Engineering, Marquette University, Milwaukee, Wisconsin, USA

\begin{abstract}
A laboratory experiment evaluated the effect of motorcycle clutch design on the electromyography (EMG) activity of the primary agonist finger flexor muscle in the forearm. The goal was to compare muscle fatigue resulting from operation of two different motorcycle clutches in simulated traffic. EMG activity from the flexor digitorum superficialis (FDS) muscle of 12 female and 11 males were recorded while each participant operated an existing motorcycle clutch (requiring $98 \mathrm{~N}$ peak force) as well as an alternate design (requiring $36 \mathrm{~N}$ peak force) during 60-minute simulations. Muscle fatigue was quantified by measuring the decrease in median frequency of the EMG signals. Compared to operating the existing clutch, male participants experienced a significant decrease in muscle fatigue between 14 to $31 \%$ when operating the alternate clutch. Females experienced a decrease of 27 to $49 \%$. In addition to reduced muscle fatigue, the alternate clutch was overwhelmingly preferred by participants and was rated superior for ease of use and comfort. Results provide a better understanding of the effect of clutch design on riders' muscular loading and implications for design improvements.
\end{abstract}

Keywords: Grip; Strength; Motorcycle; Clutch; EMG; Fatigue

Abbreviations: EMG: Electromyography; MVC: Maximum Voluntary Contraction; FDS: Flexor Digitorum Superficialis

\section{Introduction}

Motorcycle riding is a hobby commonly enjoyed by Americans with nearly 8.5 million registered motorcycles in the US [1]. Motorcycle manufacturers have enjoyed consistent increases in sales throughout the first decade of the $21^{\text {st }}$ century [2], an indication that the sport is gaining avid new riders each year. An important consideration as sales increase is the demographics of the riders themselves. Consistent with the US population, the median age of motorcycle riders has significantly increased in recent years [2]. In fact, the percent of total riders over age 40 has increased from $21.3 \%$ in 1985 to $53.0 \%$ in 2003 [2]. Thus, a concern arises when hand controls, such as those employed to operate a motorcycle, repeatedly require excessively high force levels over long periods of time during a recreational ride. Exposure to such high repetition-high force tasks has been linked to muscle fatigue, loss of productivity and an increased incidence of musculoskeletal disorders [3]. Indeed, recent studies have demonstrated the accumulation of muscle fatigue in the right forearm muscles attributed to motorcycle

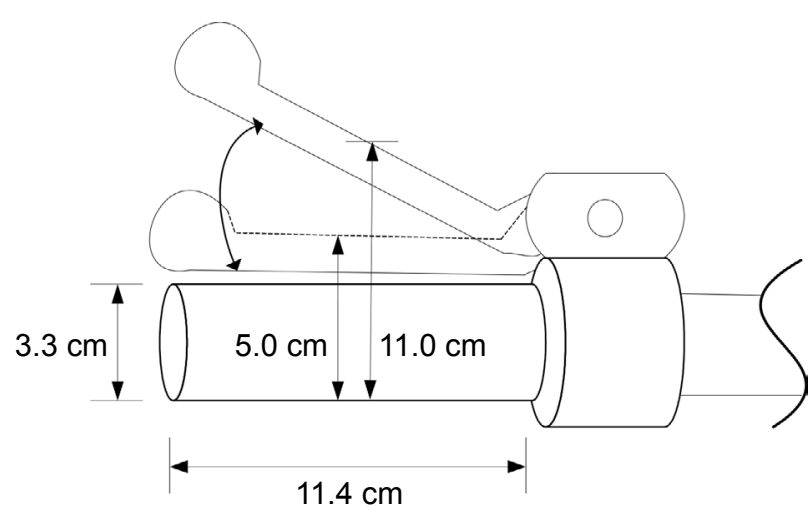

Figure 1: Diagram of clutch lever with a grip span of $11.0 \mathrm{~cm}$ measured at the midpoint of the handle. brake use [4]. Therefore, the need for a precise evaluation of motorcycle hand controls is needed as they relate to fatigue or injury to maintain safety on the roadway. Specifically, if controls such as the motorcycle clutch were redesigned to require lower force levels at adequate grip spans, it could improve the safety by minimizing fatigue.

A motorcycle clutch is controlled by a lever located on the left handlebar and is operated by left hand (Figure 1). The lever pulls on a cable connected to a hydraulic or spring-loaded clutch mechanism. Engaging and releasing the clutch handle enables a motorcycle rider to change gears while driving or at rest. The transmission is fully disengaged to the drive shaft when the lever is pulled in and engages when the lever is released. A common spring loaded clutch has a grip span of $11 \mathrm{~cm}$ (fully open) and requires up to $98 \mathrm{~N}$ of grip force to activate (Figure 1). Due to the large grip span, many individuals have difficulty adequately grasping the clutch with all 4 fingers, creating even more difficulty in exerting the high grip forces required to grasp the handle engaging the clutch.

The frequency of clutch use can increase greatly in moderate to high traffic, increasing the risk of muscle fatigue in the forearm muscles responsible for grip forces. Muscle fatigue occurs when a muscle is exerted beyond a certain level of contraction over some period of time. Physiologically, muscle fatigue is a result of several factors including an accumulation of phosphate, inhibition of the release of calcium, and a depletion of glycogen reserves to fuel the muscle [5]. The resulting effect is an inability for the muscle to continue contractions at the same level.

${ }^{*}$ Corresponding author: Megan Conrad, Department of Industrial and Systems Engineering, Oakland University, 2200 N Squirrel Rd, Rochester, MI, 48309, USA, Tel: (248) 370-4896; Fax: (248) 370-2699; E-mail: conrad@oakland.edu

Received February 28, 2014; Accepted April 21, 2014; Published April 29, 2014

Citation: Conrad MO, Marklin RW (2014) Evaluation of Forearm Muscle Fatigue from Operating a Motorcycle Clutch. J Ergonomics S4: 006. doi:10.4172/21657556.S4-006

Copyright: @ 2014 Conrad MO, et al. This is an open-access article distributed under the terms of the Creative Commons Attribution License, which permits unrestricted use, distribution, and reproduction in any medium, provided the original author and source are credited. 


\begin{tabular}{|c|c|c|}
\hline Variables & Male $\mathbf{( n = 1 0 )}$ & Female $(\mathbf{n}=\mathbf{1 2})$ \\
\hline Stature (cm) & $174.5 \pm 7.98$ & $161.2 \pm 4.87$ \\
& {$[165.6-188.5]$} & {$[153.6-168.8]$} \\
\hline Weight (N) & $818.5 \pm 172.60$ & $688.6 \pm 183.04$ \\
& {$[645.0-1068.6]$} & {$[444.8-1089.8]$} \\
\hline Hand Breadth (cm) & $8.5 \pm 0.52$ & $7.5 \pm 0.49$ \\
& {$[8.0-9.6]$} & {$[6.5-8.0]$} \\
\hline Hand Length (cm) & $18.9 \pm 1.01$ & $17.1 \pm 0.93$ \\
& {$[17.7-20.5]$} & {$[15.6-19.1]$} \\
\hline Peak Grip Strength (N) & $407.6 \pm 93.1$ & $247.9 \pm 40.9$ \\
\hline \% Maximal Grip Strength required & {$[213.6-565.2]$} & {$[186.9-322.6]$} \\
\hline to grasp and engage existing clutch (98 N) & $25 \pm 7$ & $41 \pm 7$ \\
\hline \% Maximal of Grip Strength required & $917-46]$ & {$[31-52]$} \\
to grasp and engage alternate clutch (36 N) & {$[31-52]$} & {$[11-19]$} \\
\hline
\end{tabular}

Table 1: Demographic and Anthropometric Participant Data (mean \pm sd, min $\max$.

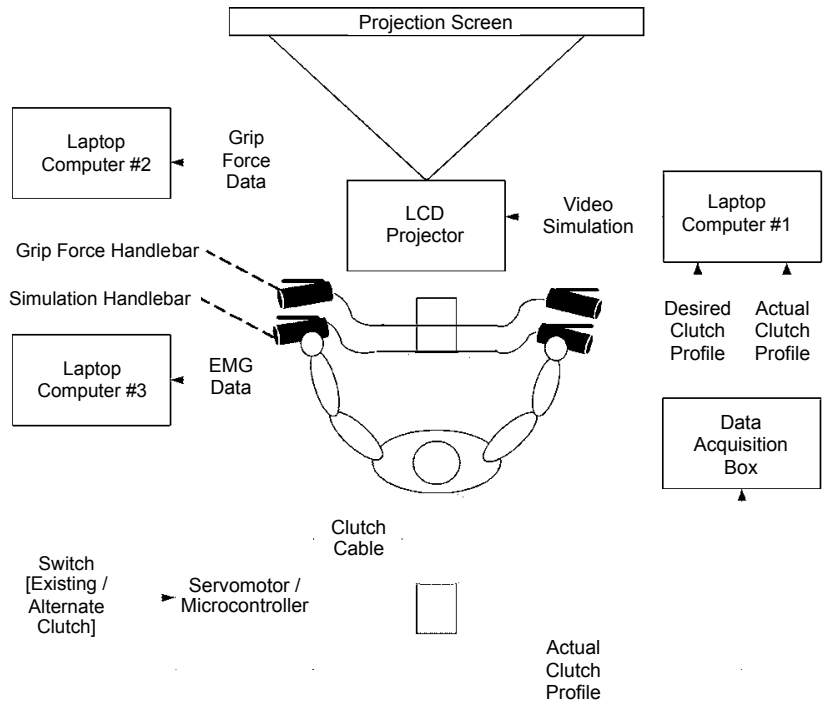

Figure 2: Schematic diagram of the laboratory set-up.

For a sustained contraction, an individual in theory can maintain $15 \%$ of a muscle's maximum voluntary contraction (MVC) for an unlimited amount of time [6]. However, many common tools and controls requiring sustained or isometric contractions exceed the $15 \%$ MVC threshold. When using these tools or controls, muscle fatigue can accumulate over time and possibly cause a decrease in strength and increase in discomfort. In some cases, such symptoms could affect an individual's ability to use the device safely.

Surface electromyography (EMG) has been used extensively to evaluate the level of fatigue in a muscle. A downward shift of median frequency of the EMG signal is one indicator of muscle fatigue [7]. The median frequency is the frequency $(\mathrm{Hz})$ from the EMG spectrum about which the power is distributed equally on either side. As the level of fatigue increases, the median frequency of the spectrum decreases linearly until exhaustion for a constant isometric contraction level [8]. The exertion level (or \%MVC of exertion) is affected by the rate of fatigue in a muscle. As a constant tension is exerted by a muscle over time (isometric), the median frequency continues to shift downward, indicating a greater level of muscle fatigue.

Median frequency analysis could be used to compare usability of 2 competing designs of a control or tool. Katsis et al. found median frequency to be a reliable indicator of muscle fatigue during driving conditions [9]. The team measured a decrease in median frequency from $9.5 \%$ to $18.9 \%$ while participants drove a truck, a tractor, and a truck with a trailer. Related to tool use, forearm muscle fatigue due to hammer use has also been studied using median frequency analysis [10], comparing muscle fatigue between hammering on a wall versus over a bench. The experimenters found that wall conditions resulted in an $8.5 \%$ higher decrease in median frequency than the bench conditions.

The purpose of this study is to evaluate muscle fatigue due to motorcycle clutch use in an existing clutch and compare the results to the fatigue produced when the same riders used an improved clutch design requiring a shorter grip span and less grip force. Evaluation of the muscle fatigue imposed by the new design versus that of the existing design provides a better understanding of the implications of the clutch and potential for future design improvements.

\section{Materials and Methods}

\section{Approach}

In a laboratory setting participants operated a motorcycle clutch with the existing (98 N peak force and $11 \mathrm{~cm}$ grip span) and alternate (36 $\mathrm{N}$ peak force ) force-displacement profiles during a 60-min simulated ride composed of a mix of typical urban riding, stop and go, and interstate traffic.

\section{Participants}

Eleven males (33.0 \pm 7.84 years) and 12 females ( $37.3 \pm 9.69$ years $)$ volunteered as participants. A statistical power test [11] indicated 9 participants of each gender were necessary to limit type I error to 0.05 and type II error to 0.20 . None of the participants reported prior upper extremity musculoskeletal disorders or injuries that may have affected performance or participants' level of discomfort. Participants had a range of riding experience and hand sizes. All participants signed a consent form approved by the Marquette University Institutional Review Board (IRB). Demographic data on the participants can be viewed in Table 1.

\section{Experimental design}

The experimental design was a mixed model. The between participant variable was gender (male or female). The within participants independent variable was clutch design (existing or alternate). The dependent variables were median frequency of the EMG signal measured from the participants' FDS muscle and subjective ratings of comfort and effort. All participants were tested using both the existing and alternate design in an alternating presentation order.

\section{Simulation apparatus}

Controlled by a servomotor, a clutch simulator was designed and built to measure the resistance force as the clutch handle was pulled in and released. The clutch simulator was a stand-alone unit that sat on the floor and had a cable attached to the motorcycle clutch. The existing and alternate force-displacement clutch profiles were programmed into the clutch simulator, enabling the use of either clutch profile on the same motorcycle. The existing clutch profile had a spring-loaded mechanism requiring a peak force of approximately $98 \mathrm{~N}$ to pull in the clutch. This is over 2.5 times greater than the force required by the alternate clutch $(36 \mathrm{~N})$.

As shown in the schematic in Figure 2, a motorcycle was mounted 


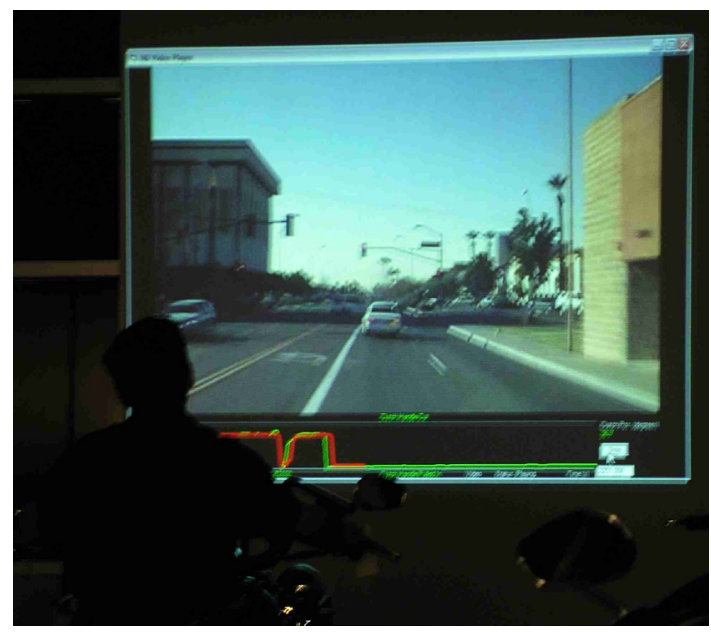

Figure 3: Participant following desired clutch profile by viewing his own clutch movement below simulation video.

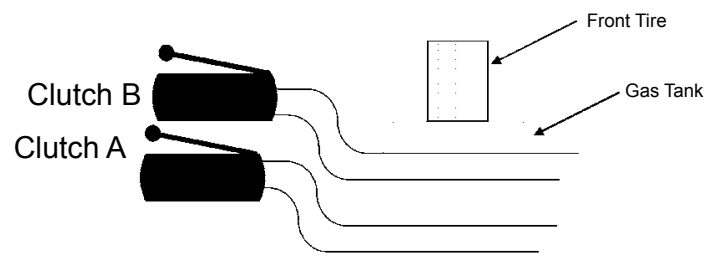

Figure 4: Orientation of Clutch A (Simulation Clutch) and Clutch B (Grip Strength Clutch) as viewed from the top of the motorcycle.

on the floor in the test room facing a projection screen approximately $3 \mathrm{~m}$ in front of the bike. Displayed on the screen was a 60-min digital video of an actual motorcycle ride conducted on streets and highways in Phoenix, AZ. The bottom of the screen displayed a scrolling signal indicating the actual angle of clutch lever measured during the ride in Phoenix (Figure 3). A potentiometer attached to the pivot point of the clutch on the motorcycle sent angular data of the lever movement to the laptop computer running the projection. As the lab clutch lever was grasped and released, the angle of the clutch handle was displayed on the bottom of the screen, thereby allowing the participant to mimic the desired clutch movement. Simultaneous to the video and clutch monitoring, a Biometrics DataLink EMG system (Biometrics Ltd., Gwent, UK) was used to record EMG data from the participant's left FDS muscle.

\section{Grip strength and sustained contraction}

An additional set of handlebars containing a "grip strength clutch" (Clutch B) was also mounted on the motorcycle, as shown in Figure 4. Before testing on the 2 clutch simulations, maximum grip strength was exerted on Clutch B for a minimum of $5 \mathrm{sec}$ while a $1000 \mathrm{lbs}$. capacity load cell (Sensotec Sensors, Columbus $\mathrm{OH}$ ) measured the amount of grip force exerted on the clutch handle. Peak grip force was calculated as the average maximum grip force exerted during the middle 4 seconds of the 5 second trial. The grip span of Clutch B was set at $6.5 \mathrm{~cm}$ for females and $8.0 \mathrm{~cm}$ for males (center of clutch handle). These grip spans corresponded to the grip span where females and males, respectively, exerted the greatest grip force in a previous study conducted in the laboratory. The load cell communicated with a customized LabVIEW (National Instruments, Austin TX) program running on a laptop computer. The program automatically calculated $60 \%$ of the participant's maximum exerted grip force (60\% MVC). After sufficient rest, the participant squeezed the Clutch B handle and exerted $60 \%$ MVC force as long as he or she could maintain the exertion. The program displayed a range of $60 \% \mathrm{MVC} \pm 22 \mathrm{~N}$ (10\% of average grip strength), and subjects were instructed to maintain grip force within this range. When the participant could no longer maintain grip force within $60 \% \mathrm{MVC} \pm 22 \mathrm{~N}$, he or she stopped gripping the handle. $60 \%$ MVC was chosen for the exertion because it was a level that could be sustained by participants for $5 \mathrm{sec}$ periods throughout the hour long simulation experiment.

\section{Electromyography}

Surface electrodes were attached to the clean and abraded left forearm skin adjacent to the FDS muscle to record muscle activity as the fingers were flexed around the clutch handle [12]. The electrodes were attached to the EMG system through a portable unit strapped around the participant's waist. This unit was connected to the laptop computer running the Biometrics Analysis Software (Biometrics Ltd., Gwent, UK). EMG signals were amplified (x1000) prior to sampling at $1000 \mathrm{~Hz}$. The system applied a digital bandpass filter $(10-350$ $\mathrm{Hz})$ targeting EMG activity and a separate notch filter $(59-61 \mathrm{~Hz})$ to eliminate line noise.

\section{Experimental protocol}

Each participant was first briefed on the experimental objectives. Their grip strength was tested using Clutch $B$ and the peak grip reading was entered into the grip strength program that calculated 60\% MVC. Surface electrodes were attached to the skin over the left FDS muscle. The experimenter checked EMG signals and entered file names on the EMG software. Time was given for the participants to sit on the motorcycle and familiarize themselves with the set-up. Participants were asked to maintain $60 \%$ MVC until exhaustion while EMG signals were recorded. One of the clutch profiles (existing or alternate) was randomly selected. The experimenter began the simulation video with the selected clutch profile (Figure 4). The participant followed the clutch profile with Clutch A. Every $5 \mathrm{~min}$ the experimenter asked the participant to switch to Clutch B to grip 60\% MVC for 5 sec while EMG signals were recorded. Upon completion of the $5 \mathrm{sec}$ reading, the participant immediately returned to Clutch A to follow the simulation on the screen. After 60 minutes the simulation video concluded. The participant was given at least 1 hour of rest time during which anthropometric measurements were taken and recorded. The participant then repeated the same procedure on the remaining clutch profile. Immediately following each simulated ride, the participants were asked to subjectively rate the alternate and existing clutches for overall comfort, ease of use and clutch rank.

\section{Data conditioning and analysis}

Musclme Fatigue: Muscle fatigue was calculated using median frequency analysis of the raw EMG data for the FDS muscle taken during the $60 \%$ MVC sustained contractions. The median frequency is defined as the frequency that divides the power spectrum into two regions having the same power, or area under the amplitude-frequency curve such that:

$$
\int_{0}^{f_{\text {med }}} S(f) d f=\int_{f_{\text {med }}}^{\infty} S(f) d f
$$

Median frequencies were calculated using the Biometrics Analysis 
Citation: Conrad MO, Marklin RW (2014) Evaluation of Forearm Muscle Fatigue from Operating a Motorcycle Clutch. J Ergonomics S4: 006. doi:10.4172/2165-7556.S4-006

Page 4 of 6

\begin{tabular}{|c|c|}
\hline Data Collection Points & $\begin{array}{c}\text { Approximate \% time clutch pulled in } \\
\text { during prior } \mathbf{5} \text { min }\end{array}$ \\
\hline $5 \mathrm{~min}$ & $62 \%$ \\
\hline $20 \mathrm{~min}$ & $50 \%$ \\
\hline $35 \mathrm{~min}$ & $70 \%$ \\
\hline $50 \mathrm{~min}$ & $58 \%$ \\
\hline $60 \mathrm{~min}$ & $70 \%$ \\
\hline
\end{tabular}

Table 2: Approximate time clutch was grasped, fully engaging the clutch, during the 5-min prior to each EMG data collection point during the 60-min simulated ride.

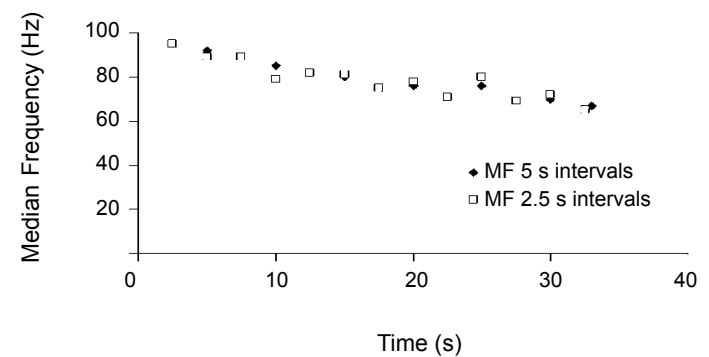

Figure 5: One participant's median frequency (MF) of flexor digitorum superficialis (FDS) over time until exhaustion.

\begin{tabular}{|c|c|c|c|c|c|}
\hline & & \multicolumn{4}{|c|}{ Percentage of Muscle Fatigue (\%) } \\
\hline & & \multicolumn{2}{|c|}{ Females $(n=12)$} & \multicolumn{2}{|c|}{ Males $(n=10)$} \\
\hline & & $\begin{array}{l}\text { Existing } \\
\text { Clutch }\end{array}$ & $\begin{array}{l}\text { Alternate } \\
\text { Clutch }\end{array}$ & $\begin{array}{c}\text { Existing } \\
\text { Clutch }\end{array}$ & $\begin{array}{l}\text { Alternate } \\
\text { Clutch }\end{array}$ \\
\hline \multirow{5}{*}{ 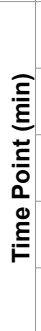 } & 5 & $\begin{array}{l}57 \% \pm 38 \\
{[0-100]}\end{array}$ & $\begin{array}{l}30 \% \pm 30 \\
{[0-100]}\end{array}$ & $\begin{array}{l}49 \% \pm 41 \\
{[0-100]}\end{array}$ & $\begin{array}{c}35 \% \pm 31 \\
{[0-87]}\end{array}$ \\
\hline & 20 & $\begin{array}{c}62 \pm 60 \\
{[0-100]}\end{array}$ & $\begin{array}{l}25 \pm 25 \\
{[0-60]}\end{array}$ & $\begin{array}{c}56 \pm 33 \\
{[0-100]}\end{array}$ & $\begin{array}{l}32 \pm 26 \\
{[0-89]}\end{array}$ \\
\hline & 35 & $\begin{array}{c}66 \pm 27 \\
{[16-100]}\end{array}$ & $\begin{array}{l}28 \pm 22 \\
{[0-61]}\end{array}$ & $\begin{array}{c}43 \pm 32 \\
{[5-100]}\end{array}$ & $\begin{array}{l}20 \pm 25 \\
{[0-76]}\end{array}$ \\
\hline & 50 & $\begin{array}{c}51 \pm 41 \\
{[0-100]}\end{array}$ & $\begin{array}{l}25 \pm 31 \\
{[0-96]}\end{array}$ & $\begin{array}{c}39 \pm 33 \\
{[0-100]}\end{array}$ & $\begin{array}{l}16 \pm 22 \\
{[0-65]}\end{array}$ \\
\hline & 60 & $\begin{array}{c}80 \pm 21 \\
{[38-100]}\end{array}$ & $\begin{array}{l}31 \pm 26 \\
{[0-78]}\end{array}$ & $\begin{array}{c}53 \pm 33 \\
{[0-100]}\end{array}$ & $\begin{array}{l}22 \pm 24 \\
{[0-78]}\end{array}$ \\
\hline
\end{tabular}

Table 3: Percentage (mean \pm sd, min - max) of FDS muscle fatigue for females and males on existing and alternate clutches at each of the 5 time points during the 60 -min simulated ride.

software from the $5 \mathrm{sec}$ raw EMG data recorded every 5 min throughout the simulated ride. Of the 13 EMG data collection points taken throughout the 60 -min simulation, 5 of these data points $(5,20,35$, 50 , and $60 \mathrm{~min}$ ) were identified as the most relevant to muscle fatigue based on the frequency of clutch action during the prior $5 \mathrm{~min}$. Table 2 shows the 5 time points and the approximate corresponding clutch usage over the $5 \mathrm{~min}$ prior to each time point. Collecting EMG data at precisely the same times throughout the video simulation ensured participants' data could be compared. Median frequency readings were obtained from each raw data, and percentage of fatigue was calculated using Equation 1.

$$
{ }_{0} \text { Fatigue }_{i, j, k}=\frac{M F_{k, \text { max }}-M F_{i, j, k}}{M F_{k, \text { max }}-M F_{k, \text { min }}}
$$

Where:

$\%$ Fatigue $_{i, j, k}=$ percentage fatigue of FDS muscle at time $i$ for participant $\mathrm{k}$ using clutch $\mathrm{j}$

$\mathrm{MF}_{\mathrm{i}, \mathrm{j}, \mathrm{k}}=\mathrm{EMG}$ median frequency of the $5 \mathrm{sec} 60 \%$ MVC exertion from participant $k$ at time $i$ using clutch $j$
$\mathrm{MF}_{\mathrm{k} \text {,min }}=\mathrm{EMG}$ median frequency at the end of the sustained $60 \%$ MVC exertion for participant $\mathrm{k}$

$\mathrm{MF}_{\mathrm{k}, \max }=\mathrm{EMG}$ median frequency at the beginning of the sustained $60 \%$ MVC exertion for participant $k$

Statistical Analysis: Statistica software (Statsoft, Tulsa, OK) was used to evaluate the main effect of gender on the percentage of fatigue in the mixed model ANOVA ( $\alpha=0.05$ ), SPSS (SPSS Inc., Chicago, IL) was used to assess main effects of clutch and gender with nonparametric tests of the ordinal subjective data.

\section{Results}

\section{Median frequency}

Median frequency was found to decrease linearly over time throughout the sustained contractions. The time to complete exhaustion (when the $60 \%$ sustained contraction could no longer be maintained) ranged from 25 to $113 \mathrm{sec}$ among participants. Figure 5 depicts a typical linear decrease in median frequency of the EMG signal over time for one participant.

\section{Muscle fatigue}

At each time point $(5,20,35,50$, and $60 \mathrm{~min})$, the mean percentage of muscle fatigue for both males and females was substantially lower when the participant used the alternate clutch vs the existing clutch. Table 3 and 4 reveal the decrease in muscle fatigue for males and females using the alternate vs the existing clutch. Figure 6 graphically depict the mean difference in percentage fatigue between the existing and alternate clutches for females and males, respectively, across all 5 time points. As shown in Table 4, the alternate clutch reduced fatigue more for females ( $27 \%$ to $49 \%$, absolute difference) than males ( $14 \%$ to $31 \%$ ) across 4 of the 5 intervals ( $\mathrm{p}$ values ranged from $<0.001$ to 0.005 ).
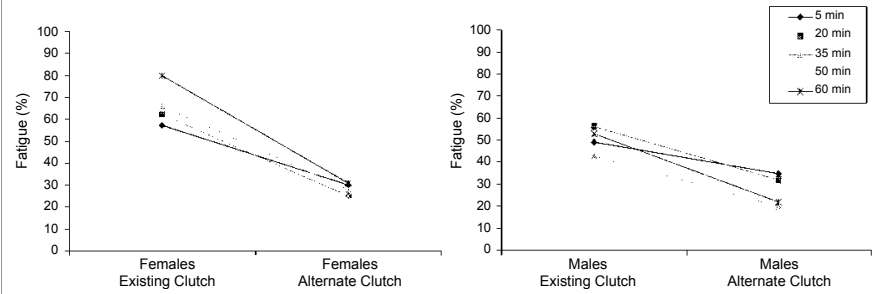

Figure 6: Mean percentage of muscle fatigue for males $(n=11)$ and females $(n=12)$ at 5 time points during the 60 -minute simulated ride.

\begin{tabular}{|c|c|c|c|c|c|}
\hline & & \multicolumn{4}{|c|}{ Percentage Difference in Fatigue $(\%)^{1}$} \\
\hline & & \multicolumn{2}{|c|}{ Females $(n=12)$} & \multicolumn{2}{|c|}{ Males $(n=10)$} \\
\hline & & $\begin{array}{l}\text { Absolute } \\
\text { Difference }\end{array}$ & $\begin{array}{l}\text { Relative } \\
\text { Decrease }\end{array}$ & $\begin{array}{l}\text { Absolute } \\
\text { Difference }\end{array}$ & $\begin{array}{c}\text { Relative } \\
\text { Decrease }\end{array}$ \\
\hline \multirow{5}{*}{ 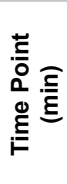 } & 5 & $27 \%^{a}$ & $47 \%$ & $14 \%{ }^{a}$ & $29 \%$ \\
\hline & 20 & $37^{\mathrm{b}}$ & 60 & $24^{b}$ & 43 \\
\hline & 35 & $38^{c}$ & 58 & $23^{c}$ & 53 \\
\hline & 50 & $26^{d}$ & 51 & $23^{d}$ & 59 \\
\hline & 60 & $49^{e}$ & 61 & $31^{\mathrm{e}}$ & 58 \\
\hline
\end{tabular}

${ }^{1}$ Difference $=(\%$ fatigue existing clutch $)-(\%$ fatigue alternate clutch $)$ ${ }^{\mathrm{a} p}=0.004$

${ }^{\mathrm{b}} \mathrm{p}<0.001$

${ }^{c} p<0.001$

${ }^{d} p=0.002$

ep $<0.001$

Table 4: Means of absolute difference and relative decrease in mean muscle fatigue of the FDS muscle between the existing and alternate clutch designs for males and females at 5 time points during the 60 -min simulated ride. 


\section{Perceived ease of use}

Ease of use was ranked on a similar scale from 1 to 7 , with 1 being 'very difficult to use' to 7 being 'very easy to use. Female participants ranked the existing clutch with a median rating of 2 , much lower (more difficult) than their median ranking of 6 for the alternate clutch $(\mathrm{p}<$ $0.05)$. Male participants rated the existing clutch with a median rating of 4 while rating the alternate clutch with a median of $6(\mathrm{p}<0.05)$.

\section{Perceived overall rating}

Participants ranked overall comfort on a scale from 1 to 5 , with 1 representing 'poor', 2 being 'fair', 3 being 'average', 4 being 'good' and 5 being 'excellent'. Female participants ranked the existing clutch with a mean comfort rating of 2 (fair), much lower than their ranking of 4 (good) for the alternate clutch $(\mathrm{p}<0.01)$. Male participants rated the existing clutch at a 3 (average), while rating the alternate clutch at a 4 (good) $(\mathrm{p}<0.01)$.

\section{Alternate versus Existing Clutch Rank}

Finally, participants ranked the clutches against each other. Each participant ranked either the alternate or existing design as the 'better' or 'worse' design. Of these rankings, 9 of 11 males and 11 of 12 females ranked the alternate design as the 'better' design.

\section{Discussion}

This purpose of this study was to evaluate an existing motorcycle clutch and compare it to a feasible, yet improved alternate design. Results show the alternate design, with a reduced activation force and smaller grip span, is superior to the existing clutch with respect to forearm fatigue of the rider. . EMG data correspond with the subjective ratings in that the alternate design was rated easier to use and superior to the existing clutch.

While EMG data could not be collected on an actual ride due to the size and nature of the equipment, the simulated motorcycle ride was set up as realistically as possible and simulated an actual motorcycle ride. The video for the simulation and the clutch movements was recorded during a motorcycle ride in Phoenix, AZ. The individual riding the motorcycle while the simulation images and clutch movements were recorded was an experienced rider and motorcycle safety instructor. Therefore, all clutch movements recorded were consistent with safe riding procedures. Furthermore, the motorcycle in the laboratory setup was the same motorcycle used when recording the actual video and clutch data in Phoenix.

The method of data recording in the laboratory minimized the influence of confounding variables and allowed for an accurate comparison of muscle fatigue between the 2 clutches. In the laboratory, the clutch movements from the actual ride were synchronized to the video and scrolled directly below the video, ensuring each participant could accurately follow the same clutch movements during the 60-minute ride in Phoenix. The participants were able to view how closely they followed the actual clutch movements and could adjust as necessary. Additionally, the effects of weather and driving style were not a factor for muscle fatigue because of the controlled indoor environment in the laboratory.

The alternate clutch design is believed to be a feasible option for motorcycles. According to grip force data provided by the study's sponsor, hydraulic clutches exist that require only $36 \mathrm{~N}$ maximum grip force, which was the peak force chosen for the alternate clutch profile in this study. A common motorcycle clutch is spring loaded because it is less expensive than hydraulic clutches. With a focus on user satisfaction, motorcycle manufacturers are now interested in clutch designs that can increase comfort for existing users and attract the interest of new customers.

Median frequency of EMG is an accepted method for determining muscle fatigue. Previously, both median frequency and mean frequency analyses were employed to estimate muscle fatigue using surface EMG data. In this study, a median frequency analysis was chosen as it has been demonstrated to be less sensitive to noise [13] while still sensitive to fatigue [14]. The linear decrease in median frequency observed throughout a sustained contraction at a constant force level is consistent with prior research [8], as is shown in Figure 5 from a participant's data. As muscle fatigue increases, the median frequency decreases due to a decreased firing rate and inability to recruit additional motor units [15]. The median frequency decreases because the firing of motor units becomes more synchronized as a muscle fatigues, thereby causing a less random firing pattern. Additionally, the fast twitch fibers in the muscle fatigue faster than slow twitch fibers, thereby reducing the high frequency component of the EMG power spectrum. The equation used to quantify the percentage of muscle fatigue has been proven successful in a prior study evaluating muscle fatigue from hammer use [10].

In subjective assessments of the 2 clutch profiles, both male and female participants ranked the alternate design as requiring substantially less physical effort. They also ranked the alternate design as being more comfortable and easier to use. However, the alternate clutch was not unanimously preferred. Two males and 1 female preferred the existing clutch over its counterpart. The 3 participants ranking the existing design as better felt there was better feedback in the existing design, allowing them to feel when the clutch was engaged. Perhaps these 3 participants, all avid experienced riders, preferred the existing clutch because they were more familiar with the existing clutch.

Muscle fatigue data indicated the alternate clutch design resulted in much less fatigue than that resulting from the existing design. As a percentage of fatigue from the existing clutch, male participants experienced an absolute difference of 14 to $31 \%$ less muscle fatigue when operating the alternate clutch at the 5 time points during the 60-min simulated ride (Table 4 and Figure 6). The decrease in fatigue from the alternate clutch was more striking for females - an absolute difference of fatigue ranging from 27 to $49 \%$ at the 5 time points. Therefore, while the alternate clutch would seem to help females greater than males, the data indicated both male and female riders would experience significantly less muscle fatigue. In addition, the alternate clutch could enhance safety and pleasure for the rider while creating a larger pool of potential riders of large motorcycles, particularly women. At least $1 / 3$ of the female participants stated that the force required to operate the clutch was a critical factor in their selection of a motorcycle model or the reason they did not ride a motorcycle.

The clutch is an integral control in the operation of a motorcycle. Reduced muscle fatigue from the alternate clutch, which requires 36 $\mathrm{N}$ grip force vs. $98 \mathrm{~N}$ for a conventional spring-loaded clutch, would in theory enable a rider to have more control over the motorcycle, particularly in urban traffic. The lower force of the alternate clutch would also enable a larger percentage of the general population to ride motorcycles that have conventional spring-loaded clutches. In addition, for those people who have forearm muscular pain or discomfort from using the conventional clutch, the alternate clutch allows the person to focus more on the riding experience and the surrounding environment and less on the pain. 
Citation: Conrad MO, Marklin RW (2014) Evaluation of Forearm Muscle Fatigue from Operating a Motorcycle Clutch. J Ergonomics S4: 006. doi:10.4172/2165-7556.S4-006

\section{Conclusion}

This study demonstrates median frequency analysis was a successful method for quantifying muscle fatigue from operating a motorcycle clutch. Compared to a typical spring-loaded clutch ( $98 \mathrm{~N}$ force), muscle fatigue was substantially less for an alternate clutch that required $36 \mathrm{~N}$, and participants overwhelmingly preferred the alternate forcedisplacement profile. This methodology could also be used to evaluate muscle fatigue from the operation of other controls for motorcycles (e.g. brake lever) and similar controls for general equipment and aid engineers in their efforts to improve the overall design of controls.

\section{Acknowledgement}

Harley-Davidson Motor Co. (Milwaukee, WI) sponsored this project.

\section{References}

1. Federal Highway Administration. U.S. Department of Transportation (2012) Highway Statistics 2012. Washington, DC

2. Morris CC (2009) Motorcycle Trends in the United States. US Department of Transportation Research and Innovative Technology Administration, Washington DC.

3. Silverstein BA, Fine LJ, Armstrong TJ (1986) Hand wrist cumulative trauma disorders in industry. $\mathrm{Br} \mathrm{J}$ Ind Med 43: 779-784.

4. Marina M, Torrado P, Busquets A, Rios JG, Angulo-Barroso R (2013) Comparison of an intermittent and continuous forearm muscles fatigue protocol with motorcycle riders and control group. J Electromyogr Kinesiol 23: 84-93.

5. Boyas S, Guevel A (2011) Neuromuscular fatigue in healthy muscle: Underlying factors and adaptation mechanisms. Ann Phys Rehabil Med 54: 88-108.
6. Rohmert W (1973) Problems in determining rest allowances. Part 1: Use of modern methods to evaluate stress and strain in static muscular work. App Ergons 4: 91-95.

7. Gonzalez-Izal M, Malanda A, Gorostiaga E, Izquierdo M (2012) Electromyographic models to assess muscle fatigue. J Electromyogr Kinesio 22: $501-512$

8. Petrofsky J, Lind A (1980) Frequency analysis of the surface electromyogram during sustained isometric contractions. Eur J Appl Physiol Occup Physiol 43 397-411.

9. Katsis CD, Ntouvas NE, Bafas CG, Fotiadis DI (2004) Assessment of muscle fatigue during driving using surface EMG. Proceedings of the IASTED International Conference on Biomedical Engineering, Innsbruck, Austria.

10. Schoenmarklin RW, Marras WS (1989) Effects of Handle Angle and Work Orientation on Hammering: II. Muscle Fatigue and Subjective Ratings of Body Discomfort. Human Factors 31: 413-420.

11. Montgomery D (2001) Design and Analysis of Experiments. John Wiley \& Sons, Inc, New York.

12. Leis AA, Trapani VC (2000) Atlas of Electromyography. Oxford University Press, New York.

13. Stulen FB, De Luca CJ (1981) Frequency parameters of the myoelectric signa as a measure of muscle conduction velocity. IEEE Transactions on Biomedical Engineering 28: 515-523

14. Kupa EJ, Roy SH, Kandarian C, DeLuca CJ (1995) Effects of muscle fiber type and size on EMG median frequency and conduction velocity. J Appl Physio 79: 23-32.

15. Basmajian JV, De Luca CJ (1985) Muscles Alive: Their Functions revealed by electromyography. Williams \& Wilkins, Baltimore.
Citation: Conrad MO, Marklin RW (2014) Evaluation of Forearm Muscle Fatigue from Operating a Motorcycle Clutch. J Ergonomics S4: 006. doi:10.4172/2165 7556.S4-006
This article was originally published in a special issue, Ergonomics and Musculoskeletal Disorder handled by Editor(s). Prof. Dr. Miguel E. Acevedo Alvarez, Chile, USA
Submit your next manuscript and get advantages of OMICS Group submissions

Unique features:

User friendly/feasible website-translation of your paper to 50 world's leading languages Audio Version of published paper

Digital articles to share and explore

Special features:

350 Open Access Journals

30,000 editorial tean

21 days rapid review proces

Quality and quick editorial, review and publication processing

Indexing at PubMed (partial), Scopus, EBSCO, Index Copernicus and Google Scholar etc

Sharing Option: Social Networking Enabled

Authors, Reviewers and Editors rewarded with online Scientific Credits

Better discount for your subsequent articles

Submit your manuscript at: www.omicsonline.org/submission 